\title{
Severe traumatic brain injury is associated with a unique coagulopathy phenotype
}

\author{
Jason M. Samuels, MD, Ernest E. Moore, MD, Christopher C. Silliman, MD, PhD, Anirban \\ Banerjee, PhD, Mitchell J. Cohen, MD, Arsen Ghasabyan, MPH, James Chandler, Julia R. \\ Coleman, MD, MPH, Angela Sauaia, MD, PhD \\ Department of Surgery (J.M.S., A.B., J.R.C.), University of Colorado Denver; Department of \\ Surgery (E.E.M., M.J.C., A.G., J.C.), Denver Health Medical Center; Department of Pediatrics \\ (C.C.S.), University of Colorado School of Public Health (A.S.), Aurora, CO.
}

\begin{abstract}
BACKGROUND: Traumatic brain injury (TBI) patients present on a spectrum from hypocoagulability to hypercoagulability, depending on the injury complexity, severity, and time since injury. Prior studies have found a unique coagulopathy associated with TBI using conventional coagulation assays such as INR; however, few studies have assessed the association of TBI and coagulopathy using viscoelastic assays that comprehensively evaluate the coagulation in whole blood. This study aims to reevaluate the TBI-specific trauma- induced coagulopathy using arrival thrombelastography. Because brain tissue is high in key procoagulant molecules, we hypothesize that isolated TBI is associated with procoagulant and hypofibrinolytic profiles compared with injuries of the torso, extremities, and polytrauma, including TBI.
\end{abstract}

METHODS: Data are from the prospective Trauma Activation Protocol study. Activated clotting time (ACT), angle, maximum amplitude (MA), 30-minute percent lysis after MA (LY30), and functional fibrinogen levels (FFLEV) were recorded. Patients were categorized into isolated severe TBI (I-TBI), severe TBI with torso and extremity injuries (TBI + TORSO/EXTREMITIES), and isolated torso and extremity injuries (I-TORSO/EXTREMITIES). Poisson regression was used to adjust for multiple confounders.

RESULTS: Overall, 572 patients (48 I-TBI, 45 TBI + TORSO/EXTREMITIES, 479 1-TORSO/ EXTREMITIES) were included in this analysis. The groups differed in INR, ACT, angle, MA, and FFLEV but not in 30-minute percent lysis. When compared with I-Torso/Extremities, after adjustment for confounders, severe I-TBI was independently associated with ACT less than 128 seconds (relative risk [RR], 1.5; 95\% confidence interval [CI], 1.1-2.2), angle less than 65 degrees (RR, 2.2; 95\% CI, 1.4-3.6), FFLEV less than 356 (RR, 1.7; 95\% CI, 1.2-2.4) but not MA less than $55 \mathrm{~mm}$, hyperfibrinolysis, fibrinolysis shutdown, or partial thromboplastin time (PTT) greater than 30 .

Address for reprints: Angela Sauaia, MD, PhD, University of Colorado School of Public Health, 13001 E. 17th Place, B119 Bldg 500, E3309 Aurora, CO 80045; email: angela.sauaia@ucdenver.edu. AUTHORSHIP

J.M.S. wrote the article. A.S. conducted the analyses. E.E.M., A.S., J.R.C., and C.S.C supervised writing the article and data interpretation. A.G. and J.R.C. accrued the data and aided in article preparation.

Portions of this article was presented in the form of an oral presentation at the 13th Annual Academic Surgical Congress, January 30February 1, 2018 in Jacksonville, Florida. 
CONCLUSION: Severe I-TBI was independently associated with a distinct coagulopathy with delayed clot formation but did not appear to be associated with fibrinolysis abnormalities. Low fibrinogen and longer ACT values associated with I-TBI suggest that early coagulation factor replacement may be indicated in I-TBI patients over empiric antifibrinolytic therapy. Mechanisms triggering coagulopathy in TBI are unique and warrant further investigation.

\section{LEVEL OF EVIDENCE:}

Retrospective cohort study; prognostic; level III

\section{Keywords}

TBI; hyperfibrinolysis; trauma-induced coagulopathy; thrombelastography

Traumatic brain injury (TBI) remains the leading cause of death following injury in the United States with 53,000 deaths annually due to TBI. ${ }^{1,2}$ Severe TBI is thought to independently contribute to a bleeding diathesis, and multiple studies have linked these coagulation abnormalities in TBI patients to an increased morbidity and mortality. ${ }^{3-5}$ While trauma-induced coagulopathy (TIC) is strongly implicated in the pathogenesis of poor outcomes following trauma, how TBI contributes to TIC remains controversial.

Recently, several studies have questioned a link between TBI and TIC. Genét et al. ${ }^{6}$ found that the addition of other injuries with TBI led to a coagulopathy, rather than TBI itself. Similarly, Lee et al. ${ }^{7}$ found that TBI was not independently associated with coagulopathy and that TIC resulted from a combination of tissue injury and shock rather than a particular injury pattern. However, few studies have evaluated TIC secondary to TBI using modern viscoelastic assays, such as thrombelastography (TEG).

Traumatic brain injury has been associated with systemic hyperfibrinolysis based on nonspecific markers, such as fibrin degradation products. ${ }^{8-10}$ On the other hand, TBI has been linked to a higher rate of venous thromboembolic (VTE) events, with some studies finding that over $50 \%$ of TBI patients develop a deep venous thrombosis (DVT). ${ }^{11,12}$ Moreover, studies have shown that the brain tissue is rich in procoagulants, namely tissue factor, ${ }^{13,14}$ and that upon systemic release, these procoagulants trigger a hypercoagulable state. ${ }^{15-17}$

Recent investigations suggest that the fibrinolysis resistance (fibrinolytic shutdown) that develops from the postinjury hypercoagulable state is associated with a higher risk of macro and microthromboembolic events including VTE, acute lung injury, and multiple organ failure (MOF) ${ }^{18}$ Our aim was to compare coagulation phenotypes in severely injured patients with and without isolated TBI. Given the richness of procoagulants within the brain, we hypothesize that severe TBI is associated with increased clot strength and diminished fibrinolysis. 


\section{METHODS}

\section{Setting and Participants}

This is a retrospective review of our prospective Trauma Activation Protocol (TAP) database. This database includes adults (age, $\geq 18$ years) meeting criteria for trauma activation, the highest trauma designation, and for whom a TEG was obtained within 1 hour postinjury prior to any blood transfusions, at the Ernest E Moore Shock and Trauma Center at Denver Health, an American College of Surgeons verified and Colorado state certified Level I trauma center. The TAP study was approved by the Colorado Multiple Institution Review Board (13-3087) and performed under waiver of consent for emergency research with minimum risk.

\section{Participants}

The trauma activation criteria at Denver Health are: (1) Glasgow Coma Scale (GCS) score less than 9 with mechanism attributed to trauma; (2) respiratory compromise, obstruction and/or intubation with mechanism attributed to trauma; (3) any injury with systolic blood pressure (SBP) less than $90 \mathrm{~mm} \mathrm{Hg}$; (4) mechanically unstable pelvic injury; (5) penetrating injuries to the neck and/or torso with SBP less than $90 \mathrm{~mm} \mathrm{Hg}$;(6) gunshot wound penetrating the neck/torso or stab wounds to the neck, torso, or extremity proximal to elbow or knee; (7) amputation proximal to the ankle or wrist or (8) fall greater than or equal to 30 ft; (9) the emergency medicine attending or chief surgical resident suspects the patient is likely to require urgent operative intervention.

For this analysis, we included only patients with New Injury Severity Score (NISS) greater than or equal to 9. Exclusion criteria for this study were: transfers from other hospitals, known chronic liver disease, inherited coagulation dysfunction, or anticoagulant use prior to injury.

Severe TBI was defined as the maximum Abbreviated Injury Scale (AIS) score of all the AIS score of the head body region greater than 2 plus admission GCS score less than 9. ${ }^{19}$ Patients were categorized into three groups, based on injury patterns: 1) Isolated TBI (ITBI): Severe TBI plus AIS score less than 3 for all other body regions; 2) TBI with polytrauma (TBI + Torso/Extremities): Severe TBI and at least one AIS score greater than 2 for other body regions; and 3) Isolated Torso/Extremities (I-TORSO/EXTREMITIES): no severe TBI and at least one AIS score greater than 2 for other regions. Twenty-nine patients (4.8\% of the TAP cohort of 601 patients) with AIS head score of 3 or greater but with GCS score of 9 or greater and minor torso injuries could not be classified into any of the three groups and were excluded (see CONSORT diagram in Fig. 1).

\section{Procedures}

Whole blood samples were collected in citrated vacuum tubes in the field by paramedics or upon presentation to the emergency department (ED) from April 2014 to April 2018.

Citrated blood samples were analyzed using the TEG 5000 Thrombelastography Hemostasis Analyzer (Haemonetics, Niles, IL) by trained professional research assistants who provided onsite continuous coverage. The TEG tracing provides the following measurements: 
activated clotting time (ACT [sec]), angle $\left({ }^{\circ}\right)$, maximum amplitude (MA $\left.[\mathrm{mm}]\right)$, and percent lysis at 30 minutes after MA (LY30 [\%]). Activated clotting time and angle represent clot formation while MA correlates with clot strength. Lastly, LY30 provides a measure of fibrinolysis (clot dissolution). In addition, we obtained the functional fibrinogen levels (FFLEV), which has been shown to correlate well with the standard Von Clauss fibrinogen assay. ${ }^{20}$ Conventional coagulation assays were obtained simultaneously with TEGs. TEG values indicative of coagulopathy were defined using previously defined cutoffs as: ACT $>128$ seconds, $\mathrm{MA}<55 \mathrm{~mm}$, angle $<65$ degrees. ${ }^{21}$ To determine fibrinolysis phenotypes, we used cutoffs derived for this specific population as follows: fibrinolysis shutdown (SD) LY30 $<0.6 \%$, or and hyperfibrinolysis (LY30, 27.7). ${ }^{23}$ The cutoff for FFLEV was less than 356 $\mathrm{mg} / \mathrm{dL} .{ }^{22}$ Cutoffs for conventional coagulation assays were: prothrombin time/international normalized ratio (INR) greater than 1.3 and activated partial thromboplastin time (aPTT) > 30 seconds. $^{23}$

\section{Outcomes and Covariates}

The primary outcomes were abnormal TEG and conventional coagulation assay (INR, aPTT) values, while secondary outcomes included ventilator- and intensive care unit-free days, symptomatic VTE, and mortality. ${ }^{24}$ According to the guidelines by the American College of Chest Physicians our institution does not conduct routine VTE surveillance in trauma patients, except for those at high risk for VTE (e.g., pelvic factures and head injuries). ${ }^{25}$

\section{Statistical Analysis}

Univariate analysis was conducted using $t$ tests, Wilcoxon tests, analysis of variance or Kruskal-Wallis for numeric variables depending on whether the variable was normally distributed or skewed. Chi-square and Fisher exact tests were used for categorical variables. Multivariate analysis was conducted using Poisson regression with robust standard errors. The risk was expressed in relative risk with 95\% confidence intervals (CI). Multicollinearity was tested with variance inflation factor, which was always less than 1.2 (i.e., not important) with the dichotomized TEG values as well as conventional coagulation tests (INR and aPTT) as the dependent variables and a three-category dummy variable denoting injury patterns (with I-TBI as the reference group) as the effect of interest. The potential confounders (age, sex, body mass index $[\mathrm{BMI}]>30 \mathrm{~kg} / \mathrm{m}^{2}$, blunt mechanism, NISS, arrival SBP, time from injury to hospital arrival, volume of crystalloids prior to hospital arrival) were chosen based on previous evidence of association with TIC and/or statistically significant association in univariate analysis. ${ }^{26-29}$

We also performed a statistical decomposition, which allows the investigator to assess the contribution of individual confounders to an association of interest. For this analysis, we limit the comparison to only the categories I-TBI and I- TORSO/EXTREMITIES (thus excluding the mixed category TBI + TORSO/EXTREMITIES). We conducted two types of decompositions, namely a Blinder-Oaxaca decomposition (BOD) and a propensity score matching (PSM) for the TEG values with a significant difference by injury pattern category. 
The BOD method, widely used in econometrics, has been underused in surgery research.

${ }^{30,31}$ In brief, this regression- based decomposition method partitions the gap in an outcome of interest between two groups into "explained" (part of the gap defined by the covariates) and "unexplained" (part of the gap not accounted for any of the include covariates) portions. For example, in the case of the gap in wages between men and women, variables such as age, income, and education explained some of the wage gap, while some of it remained unexplained, which was attributed to discrimination. In the present study, we used this technique to examine whether variables known to affect coagulation tests (such as NISS, SBP, etc.) explained the gap in TEG values between patients with and without severe TBI. For the BOD decomposition, TEG values were analyzed as continuous. Specifically, for LY30, given substantial skewness, we used a log transformation (adding 0.01 to avoid zero values) to approximate normality. The other TEG values (ACT, MA, and angle) had a close to normal distribution and did not require transformations.

For the PSM decomposition, we developed a propensity score for I-TBI using a logistic regression model with the following variables: age, sex, BMI greater than $30 \mathrm{~kg} / \mathrm{m}^{2}$, blunt mechanism, NISS, admission SBP, hemoglobin, time from injury to ED, and volume of crystalloids (ml) received prior to hospital arrival. We matched I-TBI patients to up to two nearest neighbors I-TORSO/EXTREMITIES within a maximum caliper of 0.15 . We then compared the difference between I-TBI AND I- TORSO/EXTREMITIES in TEG values in the original dataset and in the matched dataset. The portion of the difference that is diminished in the matched dataset is therefore "explained" by the matching variables. It should be noted that, compared with the PSM method, BOD allows the use of the entire sample and the assessment of the influence of each individual covariate on the gap between the groups, as opposed to PSM, in which we can only evaluate the collective role of the covariates.

Numerical variables were expressed as median (lower quartile-upper quartile). All statistical tests were two-tailed with significance declared at $p$ value of 0.05 or less. Statistical analysis was conducted using SAS version 9.4 (SAS Inc., Cary, NC) and Stata (StataCorp LLC, College Station, TX).

\section{Power Analysis}

Assuming $80 \%$ power and $95 \%$ confidence, the sample was powered to detect a minimum absolute difference of 19 percentage points in the incidence of abnormal TEG values between the I-TBI and I-TORSO/EXTREMITIES groups (PASS 14 - Power Analysis and Sample Size Software (2015), NcSS, LLC. Kaysville, UT, ncss.com/software/pass).

\section{RESULTS}

A total of572 patients were included, of whom, 48 (8.4\%) were classified as I-TBI, 45 (7.9\%) as TBI + TORSO/EXTREMITIES, and $479(83.7 \%)$ as I-TORSO/EXTREMITIES. The characteristics and outcomes of the study sample by injury pattern are shown in Table 1. The three groups had similar age and sex distribution. Blunt mechanism was more frequent in the two TBI groups, and I-TORSO/EXTREMITY patients were less severely injured and had a lower mortality. The incidence of symptomatic VTE was not significantly different 
between the three groups, although it was higher in patients in the TBI+ TORSO/ EXTREMITIES group.

\section{Coagulopathy as Measured by TEG and Conventional Coagulation Tests}

The three groups differed in ACT, angle, MA, FFLEV, INR, and aPTT but not in LY30 (Table 1). Multivariate models (Table 2) confirmed that severe TBI was independently associated with an abnormal ACT (RR, 1.53; 95\% CI, 1.08-2.16), angle (RR, 2.22; 95\% CI, 1.38-3.56), FFLEV (RR, 1.67; 95\% CI, 1.16-2.39), and aPTT (RR, 1.70; 95\% CI, 1.302.23) but not with abnormal MA, hyperfibrinolysis or fibrinolytic shutdown.

\section{Decomposition of the Difference in ACT, Angle, FFLEV and aPTT Between I-TBI and I- TORSO/EXTREMITIES by the BOD Method}

Table 3 shows the BOD for abnormal ACT, angle, FFLEV and aPTT. The variables with a negative percentage indicate that, were the I-TBI and I-TORSO/EXTREMITIES groups identical regarding that variable's value, the gap would be even larger. In contrast, positive percentages indicate that, had I-TBI and I- TORSO/EXTREMITIES groups been identical regarding that variable's value, the gap would be smaller, i.e., these variables explain some of the gap. For example, if both groups had the same incidence of blunt injuries, the gap in abnormal ACT between I-TBI and I-TORSO/EXTREMITIES would be reduced by 5.3 percentage points or in other words, the difference in blunt injury rates among the two injury patterns explained 5.3 percentage points of the difference in abnormal ACT. Overall, the confounders explained less than five percentage points of any of the differences, suggesting factors other than these variables explained the differences between the two groups.

\section{Decomposition of the Difference in ACT, Angle, and LY30 Between I-TBI and I-TORSO/ EXTREMITIES by the PSM Method}

The PS model had excellent discrimination (area under the receiver-operating characteristics curve, $0.84 ; 95 \%$ CI, 0.77-0.91). Matching by the PS resulted in comparable groups regarding the covariates in the PS model (Table 4). Overall, the propensity score including the above-mentioned covariates did not explain any part of the difference in the incidence of abnormal ACT, angle, FFLEV or aPTT (Fig. 2). Indeed, the gaps in matched patients were even larger, further suggesting that minimal or none of the disparity was due to the confounders accounted for in this analysis.

\section{DISCUSSION}

Our study aimed to define the association between TBI and TIC by evaluating the association of TBI with the multiple stages of clot formation and degradation. Similar to prior studies which used prothrombin and partial thromboplastin time, this study found that TBI is associated with abnormalities in clot formation as indicated by an abnormal ACT and angle. The association with an abnormal angle may be explained by a difference in fibrinogen levels, as isolated TBI was also associated with an abnormal FFLEV. However, while the abnormal angle is most often associated with fibrinogen levels, other contributing factors to the TEG angle in the setting of TBI such as FXIII levels and clot altering metabolites remain largely unexplored. ${ }^{20}$ 
Importantly, while previous studies that linked TBI with hyperfibrinolysis used older, less comprehensive assays, this study demonstrated that the hyperfibrinolytic phenotype was found in a minority of patients with I-TBI and in those with TBI and other injuries. Thus, we found no evidence that TBI contributes to a hyperfibrinolytic phenotype. Both decompositions suggested that the effect of TBI in the abnormal TEG values was largely independent of the assessed covariates. While it is conceivable that unmeasured confounding may be at play, it is likely that a substantial part of the TBI-associated effect is due to the TBI itself and not due to confounding.

Mechanistically, how TBI contributes to these coagulation phenotypes remains unclear. The coagulopathy associated with TBI has been attributed to a multitude of causes including platelet dysfunction, endothelial activation, and inflammatory mediators. ${ }^{32}$ The most oft touted explanation of TBI's coagulation phenotype is the systemic release of brain-derived tissue factor following injury, resulting in the initiation and resultant consumption of clotting factors. ${ }^{33}$ The central nervous system is especially rich in tissue factor, and following injury and disruption of the blood brain barrier, tissue factor is released into the systemic circulation. ${ }^{34,35}$ However, one would expect a consumptive mechanism, such as that associated with tissue factor release, would trigger a hypercoagulable state in the early part following injury. Therefore, while this consumptive process is congruent to findings presented here, such a rapid consumption of clotting factors capable of triggering alterations in clotting in a matter of an hour or less is somewhat unexpected.

A separate possibility is the activation of Protein $\mathrm{C}$, a potent regulator of coagulation and fibrinolysis. Activated protein $\mathrm{C}$ is known to inactivate plasminogen activator inhibitor 1 (PAI-1), triggering a hyperfibrinolytic state. ${ }^{36}$ A study by Cohen et al., ${ }^{3}$ however, found that without tissue hypoperfusion, the severity of TBI was not associated with differences in activated protein $\mathrm{C}$ levels. Other studies have found a similar association with hypoperfusion and coagulopathy. ${ }^{37}$ This raises the potential that in many TBI patients with other injuries, hypoperfusion and shock drive coagulopathy and increase the risk of poor outcomes more than TBI itself.

One animal study using a murine model found that tissue plasminogen activator (tPA) and urokinase plasminogen activator, both proteases that cleave plasminogen into plasmin, are upregulated in TBI; however, they found that peaks of each occurred at different times postinjury. Specifically, Hijazi et al. ${ }^{38}$ found that tPA peaked immediately postinjury and then rapidly decreased, whereas urokinase plasminogen activator experienced a delayed and slower increase with maximum plasma concentrations occurring at 9 hours after injury. This study supports a growing belief that fibrinolysis after TBI is time-dependent, and that interventions aimed at treating TBI-associated fibrinolytic dysregulation should consider these time-dependent changes.

Overall, this finding has widespread clinical implications, particularly since management of TIC in a TBI patient, from anticoagulation to antifibrinolytics, remains controversial. Although the CRASH-2 study found a survival benefit with tranexamic acid (TXA) in the first 3 hours with no increased risk of complications, ${ }^{39}$ a nested analysis of this large multinational trial showed no benefit associated with TXA among patients with TBI. ${ }^{40}$ Our 
findings suggest that the great majority of TBI patients would not benefit from an antifibrinolytic agent such as TXA. However, this study failed to find an association with TBI and VTE events. This is possibly a reflection of the lack of routine surveillance for subclinical VTE events at this institution across all trauma patients. Still, other studies which used surveillance methods and larger national studies that used an aggregate of TBI patients across many centers both demonstrated an association between TBI and VTE events. ${ }^{12,41,42}$ Given the intrinsic risk of TBI for VTE, further inhibition of clot degradation via an antifibrinolytic is likely to enhance the risk and with little benefit given the lack of an association between TBI and hyperfibrinolysis.

Given these findings, a more effective approach may be to condition the start of antifibrinolytic therapies to the results of the viscoelastic assays. Indeed, our group has previously shown in a randomized clinical trial the effectiveness of TEG-driven resuscitation. ${ }^{43}$ Importantly, this study used LY30 cutoffs that differed from those previously reported. ${ }^{22}$ New cutoffs were defined using a larger cohort with the same methods as previously used, albeit without the exclusion of mildly injured patients (NISS $<15$ ).

Although many centers do not use TEG, and oftentimes a patient's condition mandates the use of empiric therapies, the finding that TBI is associated with both an abnormal angle and hypofibrinogenemia suggest early empiric administration of cryoprecipitate may prove to be an effective measure to treat TIC in patients with isolated, severe TBI. Currently, cryoprecipitate is infrequently used in the resuscitation of severely injury trauma patients. The PROMTT study demonstrated such low utilization and was unable to demonstrate an association between cryoprecipitate and mortality likely due to the small sample size. ${ }^{44}$ Indeed, the MATTERs II study found an association with severely injured patients receiving cryoprecipitate and improved survival. ${ }^{45}$ To date, the effect of early administration of cryoprecipitate in TBI patients on postinjury adverse outcomes have not been evaluated. Our findings suggest that a prospective study evaluating the use of cryoprecipitate in TBI is warranted.

This analysis contains several limitations. First, while the multivariate analysis attempts to overcome the most significant confounding variables such as degree of shock and injury severity as well as time from injury to hospital arrival, unmeasured confounders may account for differences between groups. Second, we used different cutoffs than previously described, ${ }^{22}$ which were derived for patients with Injury Severity Score greater than 15. Here, we instead used cutoffs derived from patients regardless of Injury Severity Score; however, when conducting the same analysis using the previous cutoffs, the results were remarkably similar (data not shown). Finally, the effects of coagulopathy may differ between different intracranial injury patterns (i.e., subdural versus intraparenchymal hemorrhage as well as violation of the blood-brain barrier). We are currently working on documenting the different types of TBI in the TAP database.

\section{CONCLUSION}

Overall, using viscoelastic measures of clot formation, we found that isolated TBI is independently associated with abnormalities in the rapidity of clot initiation, fibrin cross- 
linking, and hypofibrinogenemia as well as deficiencies in the intrinsic pathway, but no abnormalities in fibrinolysis. These patients may benefit from early replacement of fibrinogen. With the use of TEG or other viscoelastic coagulation point-of-care measures, we can ultimately provide individually tailored, effective hemostatic therapy for severe TBI patients.

\section{ACKNOWLEDGMENTS}

Research reported in this publication was supported by the National Institute of General Medical Sciences of the National Institutes of Health (T32 GM008315 and P50 GM049222), The National Heart, Lung, and Blood Institute (UM 1HL 120877) and the Department of Defense (USAMRAA, W81XWH-12-2-0028). The content is solely the responsibility of the authors and does not necessarily represent the official views of the National Institutes of Health (or other sponsors of the project).

Financial Disclosures: The authors appreciate research support from Haemonetics with shared intellectual property.

\section{REFERENCES}

1. Coronado VG, Xu L, Basavaraju SV, McGuire LC, Wald MM, Faul MD, Guzman BR, Hemphill JD. Centers for disease C, prevention. Surveillance for traumatic brain injury-related deaths-United States, 1997-2007. MMWR Surveill Summ. 2011;60(5):1-32.

2. Stewart RM, Myers JG, Dent DL, Ermis P, Gray GA, Villarreal R, Blow O, Woods B, McFarland M, Garavaglia J, et al. Seven hundred fifty-three consecutive deaths in a level I trauma center: the argument for injury prevention. J Trauma. 2003;54(1):66-70; discussion -1. [PubMed: 12544901]

3. Cohen MJ, Brohi K, Ganter MT, Manley GT, Mackersie RC, Pittet JF. Early coagulopathy after traumatic brain injury: the role of hypoperfusion and the protein C pathway. J Trauma. 2007;63(6): 1254-1261; discussion 1261-2. [PubMed: 18212647]

4. Epstein DS, Mitra B, O'Reilly G, Rosenfeld JV, Cameron PA. Acute traumatic coagulopathy in the setting of isolated traumatic brain injury: a systematic review and meta-analysis. Injury. 2014;45(5): 819-824. [PubMed: 24529718]

5. Kunio NR, Differding JA, Watson KM, Stucke RS, Schreiber MA. Thrombelastography-identified coagulopathy is associated with increased morbidity and mortality after traumatic brain injury. Am J Surg. 2012; 203(5):584-588. [PubMed: 22425448]

6. Genet GF, Johansson PI, Meyer MA, Sølbeck S, Sørensen AM, Larsen CF, Welling KL, Windeløv NA, Rasmussen LS, Ostrowski SR. Trauma-induced coagulopathy: standard coagulation tests, biomarkers of coagulopathy, and endothelial damage in patients with traumatic brain injury. J Neurotrauma. 2013; 30(4):301-306. [PubMed: 23134525]

7. Lee TH, Hampton DA, Diggs BS, McCully SP, Kutcher M, Redick BJ, Podbielski J, Cotton BA, Cohen M, Schreiber MA. Traumatic brain injury is not associated with coagulopathy out of proportion to injury in other body regions. J Trauma Acute Care Surg. 2014;77(1):67-72; discussion 72. [PubMed: 24977757]

8. Goodnight SH, Kenoyer G, Rapaport SI, Patch MJ, Lee JA, Kurze T. Defibrination after brain-tissue destruction: a serious complication of head injury. N Engl J Med. 1974;290(19):1043-1047. [PubMed: 4821906]

9. Kushimoto S, Shibata Y, Yamamoto Y. Implications of fibrinogenolysis in patients with closed head injury. J Neurotrauma. 2003;20(4):357-363. [PubMed: 12866815]

10. Tian HL, Chen H, Wu BS, Cao HL, Xu T, Hu J, Wang G, Gao WW, Lin ZK, Chen SW. D-dimer as a predictor of progressive hemorrhagic injury in patients with traumatic brain injury: analysis of 194 cases. Neurosurg Rev. 2010;33(3):359-365; discussion 365-6. [PubMed: 20349100]

11. Geerts WH, Code KI, Jay RM, Chen E, Szalai JP. A prospective study of venous thromboembolism after major trauma. N Engl J Med. 1994;331(24): 1601-1606. [PubMed: 7969340]

12. Van Gent JM, Bandle J, Calvo RY, Zander AL, Olson EJ, Shackford SR, Peck KA, Sise CB, Sise MJ. Isolated traumatic brain injury and venous thromboembolism. J Trauma Acute Care Surg. 2014;77(2):238-242. [PubMed: 25058248] 
13. Drake TA, Morrissey JH, Edgington TS. Selective cellular expression of tissue factor in human tissues. Implications for disorders of hemostasis and thrombosis. Am J Pathol 1989;134(5):10871097. [PubMed: 2719077]

14. Østerud B, Bjørklid E. Sources of tissue factor. Semin Thromb Hemost. 2006;32(1):11-23. [PubMed: 16479458]

15. Bach R, Gentry R, Nemerson Y. Factor VII binding to tissue factor in reconstituted phospholipid vesicles: induction of cooperativity by phosphatidylserine. Biochemistry. 1986;25(14):4007-4020. [PubMed: 3527261]

16. Giesen PL, Rauch U, Bohrmann B, Kling D, Roqué M, Fallon JT, Badimon JJ, Himber J, Riederer MA, Nemerson Y. Blood-borne tissue factor: another view of thrombosis. Proc Natl Acad Sci USA. 1999;96(5):2311-2315. [PubMed: 10051638]

17. Tian Y, Salsbery B, Wang M, Yuan H, Yang J, Zhao Z, Wu X, Zhang Y, Konkle BA, Thiagarajan P, et al. Brain-derived microparticles induce systemic coagulation in a murine model of traumatic brain injury. Blood. 2015;125(13):2151-2159. [PubMed: 25628471]

18. Meizoso JP, Karcutskie CA, Ray JJ, Namias N, Schulman CI, Proctor KG. Persistent fibrinolysis shutdown is associated with increased mortality in severely injured trauma patients. J Am Coll Surg. 2017;224(4):575-582. [PubMed: 28017804]

19. Foreman BP, Caesar RR, Parks J, Madden C, Gentilello LM, Shafi S, Carlile MC, Harper CR, Diaz-Arrastia RR. Usefulness of the abbreviated injury score and the Injury Severity Score in comparison to the Glasgow Coma Scale in predicting outcome after traumatic brain injury. J Trauma. 2007; 62(4):946-950. [PubMed: 17426553]

20. Harr JN, Moore EE, Ghasabyan A, Chin TL, Sauaia A, Banerjee A, Silliman CC. Functional fibrinogen assay indicates that fibrinogen is critical in correcting abnormal clot strength following trauma. Shock. 2013;39(1): 45-49. [PubMed: 23247121]

21. Einersen PM, Moore EE, Chapman MP, Moore HB, Gonzalez E, Silliman CC, Banerjee A, Sauaia A. Rapid thrombelastography thresholds for goal-directed resuscitation of patients at risk for massive transfusion. J Trauma Acute Care Surg. 2017;82:114-119. [PubMed: 27805995]

22. Moore HB, Moore EE, Gonzalez E, Chapman MP, Chin TL, Silliman CC, Banerjee A, Sauaia A. Hyperfibrinolysis, physiologic fibrinolysis, and fibrinolysis shutdown: the spectrum of postinjury fibrinolysis and relevance to antifibrinolytic therapy. J Trauma Acute Care Surg. 2014;77(6):811817; discussion 7. [PubMed: 25051384]

23. Stettler GR, Moore EE, Moore HB, Nunns GR, Silliman CC, Banerjee A, Sauaia A. Redefining Post Injury Fibrinolysis Phenotypes Using Two Viscoelastic Assays. J Trauma Acute Care Surg. 2018 Epub ahead of print.

24. Cohen MJ, Kutcher M, Redick B, Nelson M, Call M, Knudson MM, Schreiber MA, Bulger EM, Muskat P, Alarcon AH et al. Clinical and mechanistic drivers of acute traumatic coagulopathy. J Trauma Acute Care Surg. 2013;75(1 Suppl 1):S40-S47. [PubMed: 23778510]

25. Schoenfeld DA, Bernard GR, Network A. Statistical evaluation of ventilator- free days as an efficacy measure in clinical trials of treatments for acute respiratory distress syndrome. Crit Care Med. 2002;30(8): 1772-1777. [PubMed: 12163791]

26. Gould MK, Garcia DA, Wren SM, Karanicolas PJ, Arcelus JI, Heit JA, Samama CM. Prevention of VTE in nonorthopedic surgical patients: antithrombotic therapy and prevention of thrombosis, 9th ed: American College of Chest Physicians Evidence-Based Clinical Practice Guidelines. Chest. 2012;141(Suppl 2):e227S-e277S. [PubMed: 22315263]

27. Brown JB, Cohen MJ, Minei JP, Maier RV, West MA, Billiar TR, Peitzman AB, Moore EE, Cuschieri J, Sperry JL, et al. Characterization of acute coagulopathy and sexual dimorphism after injury: females and coagulopathy just do not mix. J Trauma Acute Care Surg. 2012;73(6):13951400. [PubMed: 23064602]

28. Moore FA, Moore EE, Sauaia A. Blood transfusion. An independent risk factor for postinjury multiple organ failure. Arch Surg. 1997;132(6):620-624; discussion 624-5. [PubMed: 9197854]

29. Sauaia A, Moore FA, Moore EE, Haenel JB, Read RA, Lezotte DC. Early predictors of postinjury multiple organ failure. Arch Surg. 1994;129(1): 39-45. [PubMed: 8279939] 
30. Balvers K, Wirtz MR, van Dieren S, Goslings JC, Juffermans NP. Risk factors for trauma-induced coagulopathy- and transfusion-associated multiple organ failure in severely injured trauma patients. Front Med (Lausanne). 2015;2:24. [PubMed: 25964951]

31. Oaxaca R Male-female wage differentials in urban labor markets. Int Econ Rev. 1973;14(3):693709.

32. Blinder AS. Wage discrimination: reduced form and structural estimates. J Hum Resour. 1973;8(4): 436-455.

33. Maegele M, Schochl H, Menovsky T, Marechal H, Marklund N, Buki A, Stanworth S. Coagulopathy and haemorrhagic progression in traumatic brain injury: advances in mechanisms, diagnosis, and management. Lancet Neurol. 2017;16(8):630-647. [PubMed: 28721927]

34. Laroche M, Kutcher ME, Huang MC, Cohen MJ, Manley GT. Coagulopathy after traumatic brain injury. Neurosurgery. 2012;70(6):1334-1345. [PubMed: 22307074]

35. Halpern CH, Reilly PM, Turtz AR, Stein SC. Traumatic coagulopathy: the effect of brain injury. J Neurotrauma. 2008;25(8):997-1001. [PubMed: 18687038]

36. Eddleston M, de la Torre JC, Oldstone MB, Loskutoff DJ, Edgington TS, Mackman N. Astrocytes are the primary source of tissue factor in the murine central nervous system. A role for astrocytes in cerebral hemostasis. J Clin Invest. 1993;92(1):349-358. [PubMed: 8326003]

37. de Fouw NJ, de Jong YF, Haverkate F, Bertina RM. Activated protein C increases fibrin clot lysis by neutralization of plasminogen activator inhibitor- no evidence for a cofactor role of protein $\mathrm{S}$. Thromb Haemost. 1988;60(2): 328-333. [PubMed: 2975409]

38. Lustenberger T, Talving P, Kobayashi L, Barmparas G, Inaba K, Lam L, Branco BC, Demetriades D. Early coagulopathy after isolated severe traumatic brain injury: relationship with hypoperfusion challenged. J Trauma. 2010;69(6):1410-1414. [PubMed: 20404754]

39. Hijazi N, Abu Fanne R, Abramovitch R, Yarovoi S, Higazi M, Abdeen S, Basheer M, Maraga E, Cines DB, Higazi AA. Endogenous plasminogen activators mediate progressive intracerebral hemorrhage after traumatic brain injury in mice. Blood. 2015;125(16):2558-2567. [PubMed: 25673638]

40. collaborators C-t, Shakur H, Roberts I, Bautista R, Caballero J, Coats T, Dewan Y, El-Sayed H, Gogichaishvili T, Gupta S, et al. Effects of tranexamic acid on death, vascular occlusive events, and blood transfusion in trauma patients with significant haemorrhage (CRASH-2): a randomised, placebo- controlled trial. Lancet. 2010;376(9734):23-32. [PubMed: 20554319]

41. Crash-2 Collaborators IBS. Effect of tranexamic acid in traumatic brain injury: a nested randomised, placebo controlled trial (CRASH-2 Intracranial Bleeding Study). BMJ. 2011;343:d3795. [PubMed: 21724564]

42. Denson K, Morgan D, Cunningham R, Nigliazzo A, Brackett D, Lane M, Smith B, Albrecht R. Incidence of venous thromboembolism in patients with traumatic brain injury. Am J Surg. 2007;193(3):380-383; discussion 383-4. [PubMed: 17320539]

43. Knudson MM, Ikossi DG, Khaw L, Morabito D, Speetzen LS. Thromboembolism after trauma: an analysis of 1602 episodes from the American College of Surgeons National Trauma Data Bank. Ann Surg. 2004;240(3):490-496; discussion 496-8. [PubMed: 15319720]

44. Gonzalez E, Moore EE, Moore HB, Chapman MP, Chin TL, Ghasabyan A, Wohlauer MV, Barnett CC, Bensard DD, Biffl WL, et al. Goal-directed hemostatic resuscitation of trauma-induced coagulopathy: a pragmatic randomized clinical trial comparing a viscoelastic assay to conventional coagulation assays. Ann Surg. 2016;263(6):1051-1059. [PubMed: 26720428]

45. Holcomb JB, Fox EE, Zhang X, White N, Wade CE, Cotton BA, del Junco DJ, Bulger EM, Cohen MJ, Schreiber MA, et al. Cryoprecipitate use in the PROMMTT study. J Trauma Acute Care Surg. 2013;75(1 Suppl 1): S31-S39. [PubMed: 23778509]

46. Morrison JJ, Ross JD, Dubose JJ, Jansen JO, Midwinter MJ, Rasmussen TE. Association of cryoprecipitate and tranexamic acid with improved survival following wartime injury: findings from the MATTERs II study. JAMA Surg. 2013;148(3):218-225. [PubMed: 23670117] 


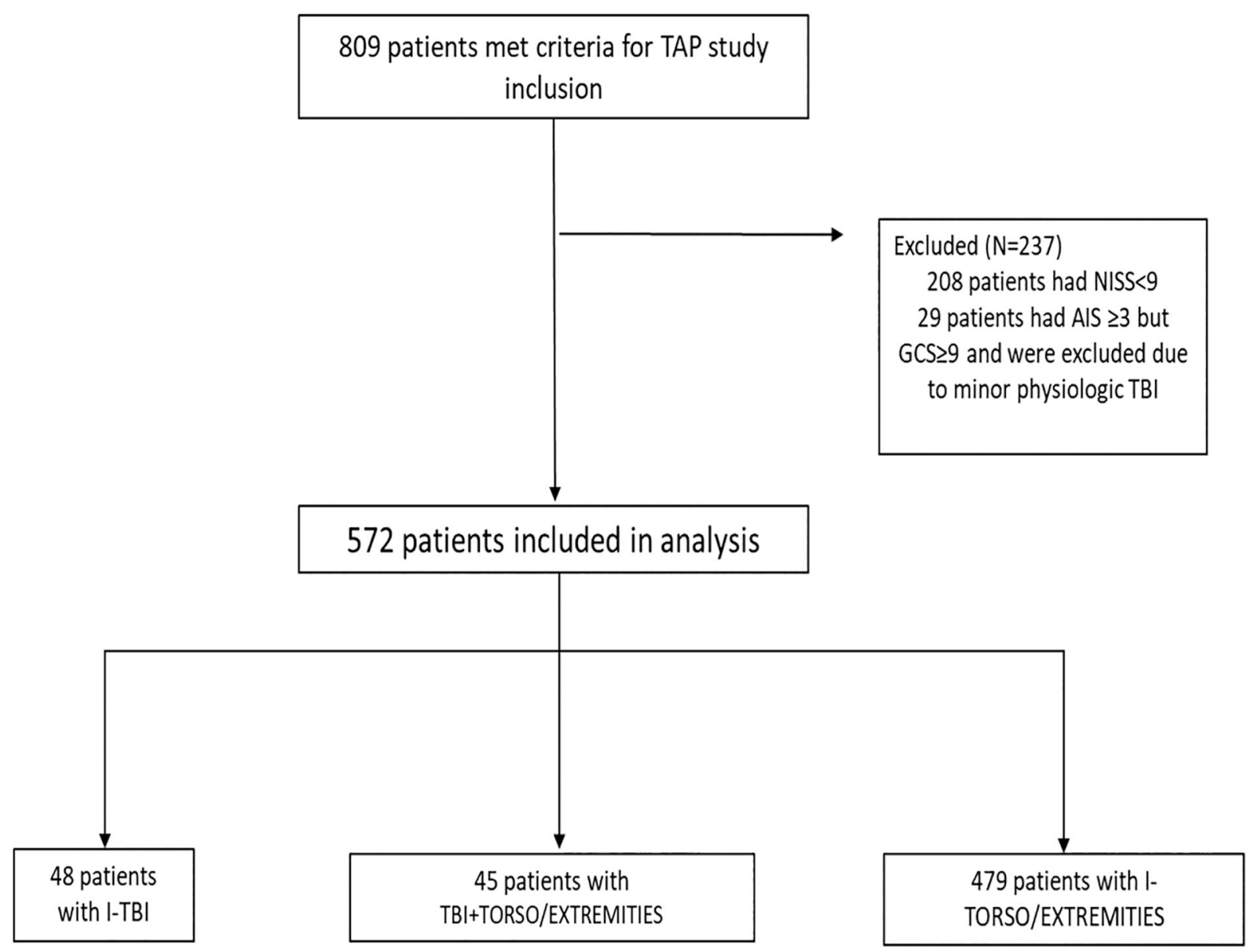

Figure 1.

CONSORT diagram. 


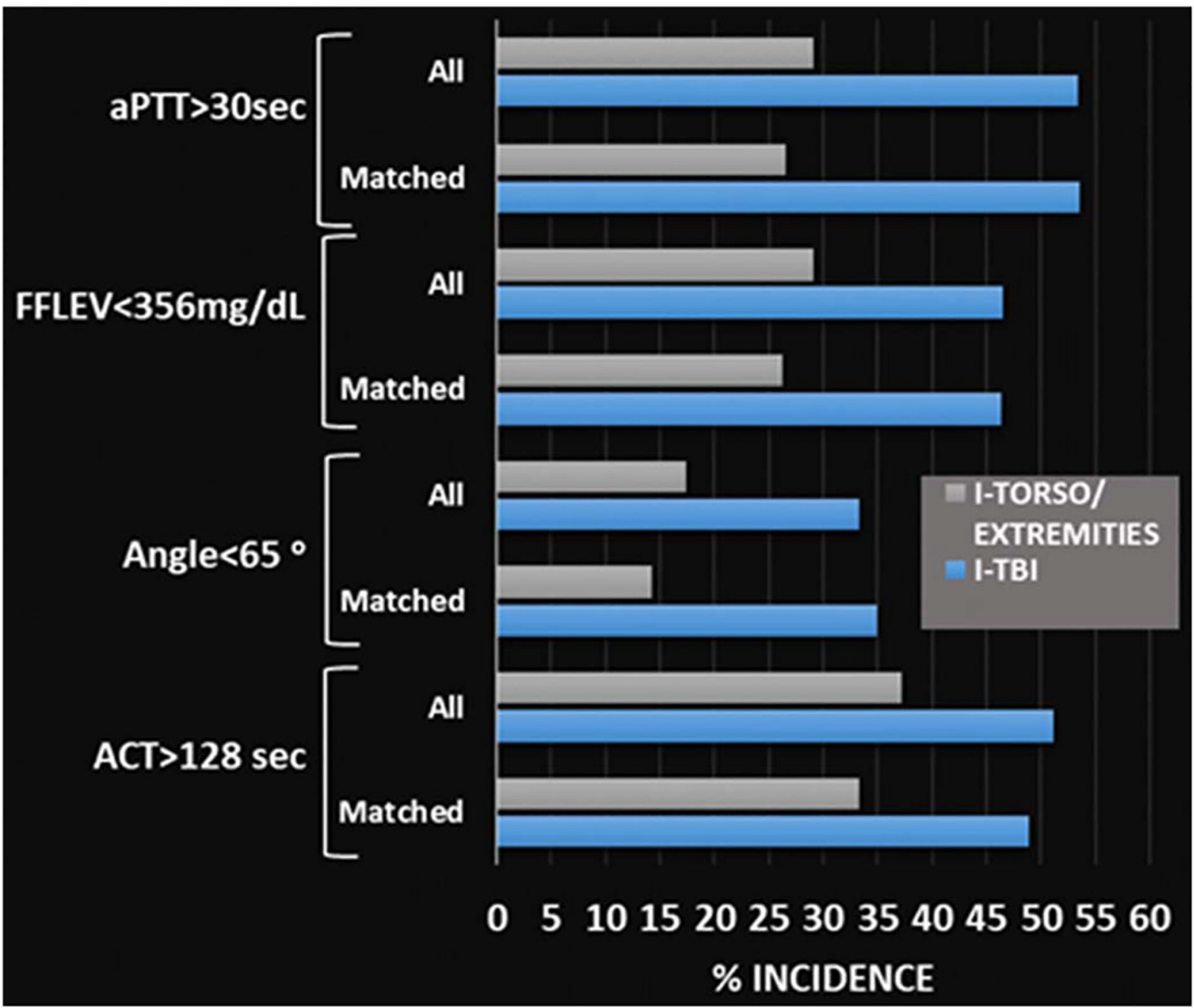

Figure 2.

Decomposition of gaps in TEG values and aPTT by PSM: difference in matched and all patients. Differences that decrease after matching are attributable to the covariates used in the PSM (age, sex, BMI > $30 \mathrm{~kg} / \mathrm{m}^{2}$, blunt mechanism, NISS, admission SBP, hemoglobin, time from injury to ED, and volume of crystalloids), while differences that do not decrease are not explained by the variables included in the PSM. 


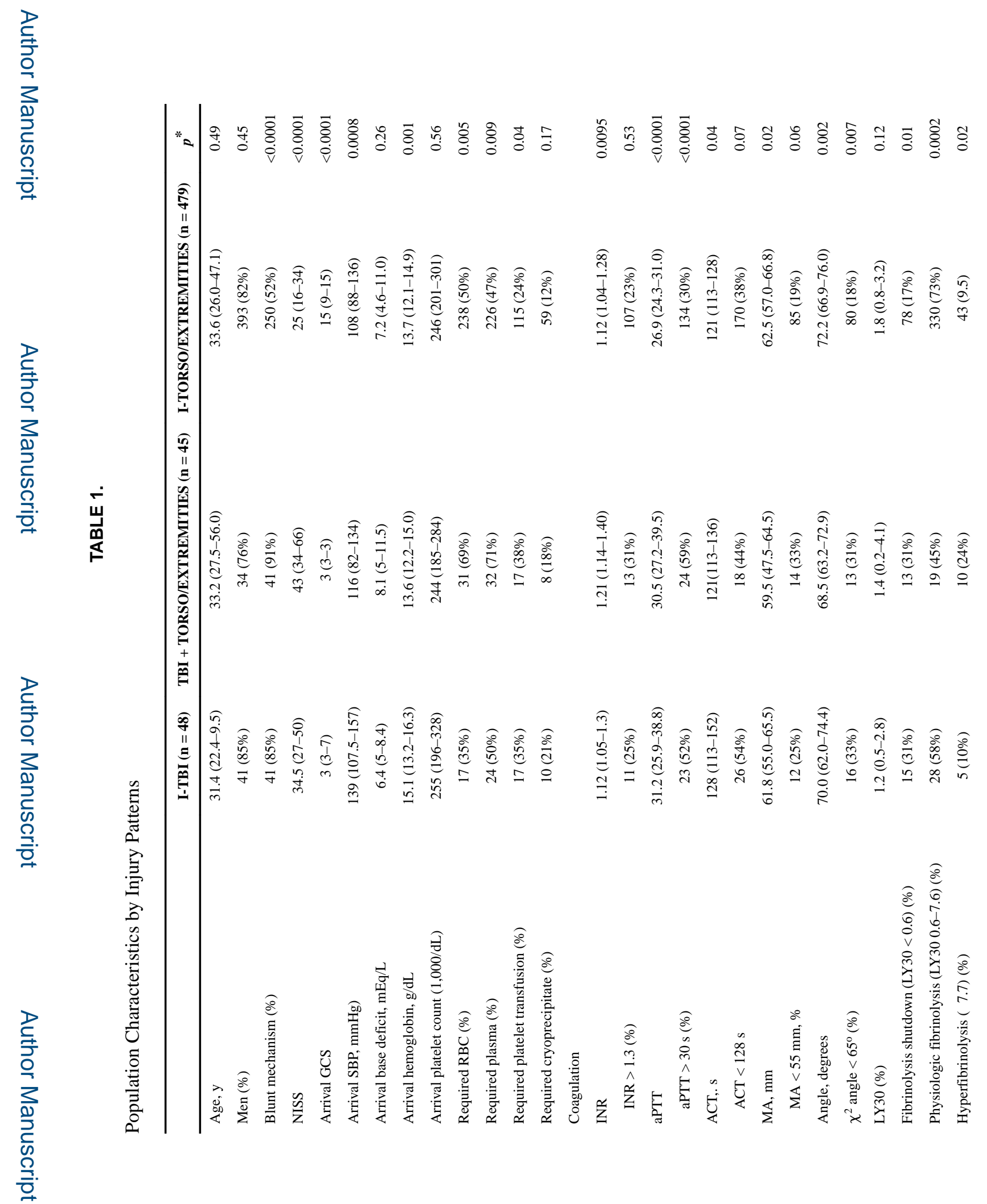

J Trauma Acute Care Surg. Author manuscript; available in PMC 2019 August 05. 
Samuels et al.

Page 15

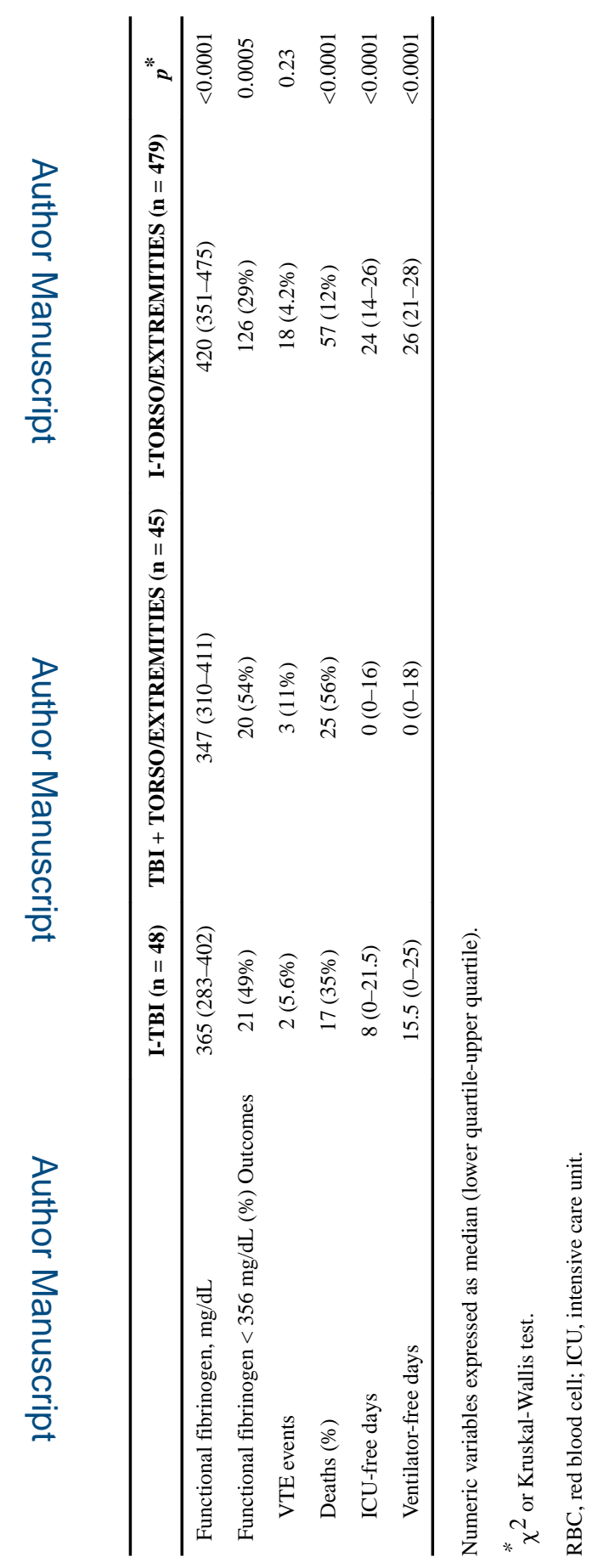

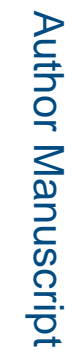




\section{TABLE 2.}

Adjusted Relative Risks of Abnormal TEG Values and Conventional Coagulation Assays in I-TBI Compared With I-TORSO/EXTREMITIES (Adjusted for Blunt Mechanism, NISS, Arrival SBP, Arrival Hemoglobin, Volume of Crystalloid Administered in the First Hour After Arrival, and Injury to Arrival Time)

\begin{tabular}{lcc}
\hline Dependent Variable & Adjusted Relative Risks (95\% CI) & $\boldsymbol{P}$ \\
\hline ACT $<128 \mathrm{~s}$ & $1.53(1.08-2.16)$ & 0.02 \\
MA $<55 \mathrm{~mm}$ & $1.69(0.97-2.98)$ & 0.07 \\
Angle $>65$ degrees & $2.22(1.38-3.56)$ & 0.0009 \\
FFLEV $<356 \mathrm{mg} / \mathrm{dL}$ & $1.67(1.16-2.39)$ & 0.006 \\
Fibrinolysis shutdown & $1.59(0.87-2.89)$ & 0.13 \\
Hyperfibrinolysis & $1.22(0.48-3.08)$ & 0.68 \\
INR & $1.45(0.87-2.41)$ & 0.15 \\
aPTT & $1.70(1.30-2.22)$ & 0.0001 \\
\hline
\end{tabular}




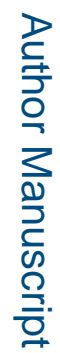

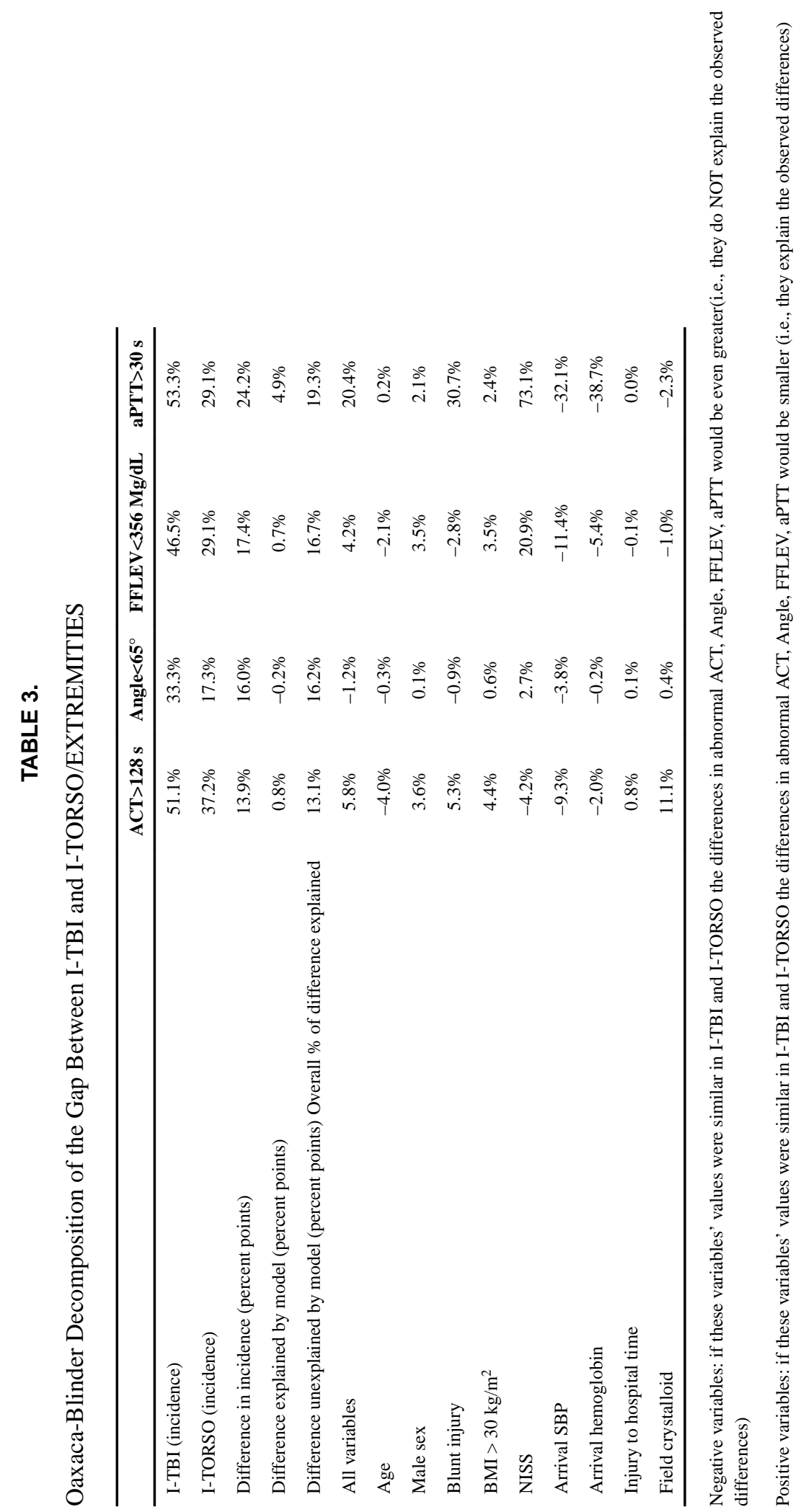


TABLE 4

Matching of I-TBI and I-TORSO/EXTREMITY Groups

\begin{tabular}{lccc}
\hline Variable & I-TBI $(\mathbf{n}=\mathbf{4 3})$ & I-Torso $(\mathbf{n}=\mathbf{8 4})$ & $\boldsymbol{p}$ \\
\hline Age, y & $30.9(21.4-52.1)$ & $35.5(26.4-49.4)$ & 0.71 \\
Male sex \% & $84 \%$ & $86 \%$ & 0.77 \\
BMI > 30 kg/m² & $16 \%$ & $12 \%$ & 0.49 \\
Percent blunt & $88 \%$ & $87 \%$ & 0.81 \\
NISS & $34(25-50)$ & $34(22-50)$ & 0.64 \\
Arrival SBP & $140(110-159)$ & $133(105-145)$ & 0.15 \\
Arrival hemoglobin & $15.0(13-16.1)$ & $15(13.4-16.2)$ & 0.85 \\
Injury to hospital arrival time, min & $24(20-35)$ & $24.5(20-34)$ & 0.98 \\
Field crystalloid, mL & $160(0-500)$ & $200(0-351)$ & 0.77 \\
\hline
\end{tabular}

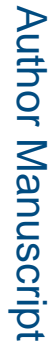

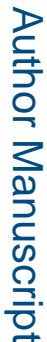

